Renal manifestation is one of the most severe complications of SLE and the clinical presentation of lupus nephritis (LN) is variable, ranging from mild asymptomatic proteinuria, renal failure to rapidly progressive glomerulonephritis. Performing renal biopsies is needed to determine the prognosis and to guide treatment in $\mathrm{LN}$.

Purpose and methods To correlate the clinical, biochemical and histopathological findings in patients with biopsy proven LN and to study the renal outcome. Retrospective analysis of 100 cases of kidney biopsy proven Lupus Nephritis was done, analysed by WHO and ISN RPS Classification of Lupus Nephritis 2003.

Results 82 were female and 18 males. Extra renal manifestations in 72 cases while rest 28 had purely renal involvement. Biopsy finding showed the following class : 8 cases of class II, 29 cases of class III, 32 cases of class IV and 3 cases of class $\mathrm{V}$. The remaining 28 showed combined class with predominant being class III+IV.

Significant microscopic hematuria, impaired GFR, proteinuria, anaemia, hypoalbuminemia, hypertension, and positive anti- dsDNA, low C3 were associated with the worst class, that is, class IV. Most of the patients with class IV or $\mathrm{V}$ had nephrotic range proteinuria and low serum albumin levels. These parameters were also correlated with high renal pathological activity and chronicity index.

24 had renal failure at the time of biopsy (mean S.creatinine $2.12 \mathrm{mg} / \mathrm{dl}$ ) followed up period of 3 years. With treatment, for 12 patients renal functions improved with creatinine improving to mean value of $1.26 \mathrm{mg} / \mathrm{dl}$. 4 patients went into severe renal failure requiring hemodialysis while 6 patients were lost to follow up.

76 patients had normal renal functions at the time of presentation with an average S.creatinine of $0.86 \mathrm{mg} / \mathrm{dl} .3$ patients went into ESRD. 15 patients lost follow up.

Conclusions Clinical, laboratory findings and renal biopsy are clinically valuable in identifying different renal classifications of lupus pathology, and to plan therapeutic strategies accordingly. LN class IV $G$ was the most common and had a reduced renal survival with significant high activity and chronicity scores.

\section{PS9:178 REMOVAL OF AN ADRENAL HORMONE ADENOMA IN A PATIENT WITH SYSTEMIC LUPUS ERYTHEMATOSUS AND AUTOMATIC FRACTURES}

G Spiliotis. Asklipieio Boulas, Boula, Greece

\subsection{6/lupus-2018-abstract.221}

Systemic lupus erythematosus is a standard autoimmune disease, characterised by the production of antibodies against cellular core. The use of corticosteroids is effective in various manifestations of lupus. We will present a 45 -year-old woman suffering from the disease for 5 years and received hydroxychloroquine, prednisolone and azathioprine. The patient received bisphosphonates and after three years had an automatic right-sided fracture of pubic bone. The patient was put up in denosumab and after one year she showed a 5th metatarsal fracture left. Computed Tomography of chest and upper abdomen were performed, where it was found an adrenal adenoma. Cortisol levels in the blood were elevated.After surgery it was found that adenoma produced cortisol. Since then, there wasn't another automatic fracture.

\section{PS9:179 THE SIDE EFFECT AND THE RETENTION RATE OF HYDROXYCHLOROQUINE IN JAPAN}

Y Ikeda, R Rokutanda, M Okada. St. Luke's International Hospital, Tokyo, Japan

\subsection{6/lupus-2018-abstract.222}

Background Hydroxychloroquine (HCQ) has been widely used in the world. However, due to the problem of retinopathy caused by chloroquine, it had not been approved in Japan until 2015, although it has been used for limited number of patients in some institutions. The primary purpose of our study is to collect the data on adverse effect of HCQ among Japanese patients with systemic lupus erythematosus. We also assessed the efficacy of our methods to improve the tolerability in cases with gastrointestinal and dermatological side effects of HCQ.

Method We retrospectively collected the data of all the 174 lupus patients treated with HCQ from the electric medical record of St Luke's International Hospital, Tokyo, Japan. We extracted the following parameters during the period between April 2008 and June 2017; patients' baseline characteristics, side effects and duration of using HCQ, and reasons of discontinuation. As for dermatological side effects, some of the patients underwent oral desensitisation; HCQ was once stopped, and after confirming negative DLST and obtaining consent from patients, it was resumed with the 36 days-desensitisation protocol. For patients with gastrointestinal side effects, some of the patients were prescribed Hangeshashinto, a traditional herbal medicine for gastrointestinal discomfort, in addition to HCQ.

Result During the period, the mean age of the patients was 41.8. 161 patients (92.5\%) were female and 152 patients $(87.4 \%)$ were treated with steroids. The average duration of using HCQ was 582.4 days. 21 patients (12.0\%) had side effects and 14 patients (8.0\%) discontinued HCQ. 10 patients had skin side effects, 7 were performed DLST, 7 were negative, and 2 resumed HCQ. 5 patients had gastrointestinal side effects, 2 were prescribed Hangeshashinto. 2 patients were possible retinopathy and stopped HCQ.

Conclusion This is one of the largest studies which reported well tolerability of HCQ in Japanese real world practice. Despite the concern of retinopathy, there were only 2 cases who stopped HCQ due to possible retinopathy in this study. In addition, our methods may help continuing HCQ in patients with skin or gastrointestinal side effects. Further larger study is needed to confirm these results.

\section{PS9:180 MANAGEMENT OF NON-RETINAL TOXICITY OF HYDROXYCHLOROQUINE}

M Okada, Masei Suda. St. Luke's International University, Tokyo, Japan

10.1136/lupus-2018-abstract.223

Although hydroxychloroquine is recommended for all the patients with systemic lupus erythematosus without contraindications, one third of lupus patients are not taking this important medication. Overcoming some of non-organ threatening side effects can lead to better drug survival rate and then improvement of overall outcome.

Since the approval of hydroxychloroquine in 2015 in Japan, we prescribed it to more than 200 patients of lupus in our institution. It is usually well-tolerated, but it is also true 
that some developed adverse effects which kept them from continuing it. As we believed that hydroxychloroquine should be continued based on the risk-benefit ratio in case of minor adverse events, such as non-severe drug eruption and gastrointestinal intolerance, we decided two systematic approaches for these problems.

For urticaria and erythematous drug rash, we first performed Drug Lymphocyte Stimulation Test (DLST), then offered oral desensitisation if the result is negative. Five patients agreed to undergo oral desensitisation over 5 weeks with crashed hydroxychloroquine tablets in conjunction with once daily levocertirizine $5 \mathrm{mg}$ at night. Desensitisation courses were started on Monday and patients were informed to contact allergologist if skin rash or pruritus emerged. All the five patients successfully went through the graded increment of hydroxychloroquine, and then continued it without the anti-histamine.

For diarrhoea, hangeshashinntou was added. Hangeshashinto, i.e. a Pinellia heart- draining decoction; in Chinese: ban xia xie xin tang), is widely administered in Japan to prevent diarrhoea induced by chemotherapy and immunosuppressive agents such as irinotecan and mycophenolate mofetil. The mechanism is reported to the inhibitory effect of glucuronidase-mediated de-conjugation. Two patients who stopped hydroxychloroquine due to diarrhoea became able to resume it with hangechashintou.

In conclusion, desensitisation of hydroxychloroquine and co-administration of hangeshashintou resulted in better tolerance of the anti-malarial in Japanese patients without serious side effects.

\section{Poster session 10: Difficult cases}

\section{PS10:182 NMDA-POSITIVE NEUROPSYCHIATRIC LUPUS MANIFESTING AS WEIGHT INCREASE AND HYPOSMIA}

${ }^{1} \mathrm{C}$ Pamfil, ${ }^{1,2} \mathrm{~S}$ Rednic, ${ }^{3}$ C Catana, ${ }^{4} \mathrm{M}$ Deac Badaranza, ${ }^{4} \mathrm{D}$ Balint Gib, ${ }^{1} \mathrm{P}$ Vele, ${ }^{5} \mathrm{~L}$ Bene, 2,4 L Damian. 'Rheumatology, Iuliu Hatieganu University of Medicine and Pharmacy, Cluj, Romania; ${ }^{2}$ Center for Connective Tissue and Musculoskeletal Diseases, Cluj, Romania; ${ }^{3}$ ENT, Sforait Medical Centre, Cluj, Romania; ${ }^{4}$ Rheumatology, Emergency Clinical County Hospital, Cluj, Romania; ${ }^{5}$ Laboratory Medicine, Emergency Clinical County Hospital, Cluj, Romania

\subsection{6/lupus-2018-abstract.224}

Background Among the clinical manifestation of systemic lupus erythematosus (SLE), neurolupus (NPSLE) is one of the hardest to identify. While active systemic diseases usually evolve with weight loss, the increase is generally associated with glucocorticoid therapy and immobilisation and rarely with hypothalamic inflammation. NMDA encephalitis, increasingly recognised, may be paraneoplastic or autoimmune.

Material and methods We present the case of a 20 year female student, with an SLE with juvenile onset, at 16, mainly with renal features and malar rash. She was in a prolonged remission on azathioprine, hydroxychloroquine, low-dose aspirin and $5 \mathrm{mg}$ prednisone/day. However, after the exam session, she came for a significant weight increment (over $12 \mathrm{~kg}$ in 2 weeks), despite allegedly normal eating, accompanied by daytime sleepiness and hyposmia. The BMI, initially 22.5, increased to 27.1 , with a $29.3 \%$ of body fat by screening impedancemetry. The analyses revealed a high ESR $(90 \mathrm{~mm} / \mathrm{h})$, an increased AAN titer (1/1280), with high anti-DNA (1055), and an anti-NMDA titer of $933 \mathrm{IU} / \mathrm{mL}$. All other tests, including lupus anticoagulant, anti-cardiolipin, and beta- 2 glicoprotein IgA, IgG, anti-Ro, anti-La, and ribosomal P antiTPO, anti-thyreoglobulin antibodies, FT4 and TSH, were normal. Cerebral MRI was unremarkable, as well as the organ involvement screening, including genital examination and PAP smear. Olfactory function measurement revealed a threshold score of 6 (normal 7-12) and an identification score of 11 (normal 12-15). She received 3 courses of methylprednisolone and cyclophosphamide courses, with slow weight decrease and normalisation after two months and some correction of hyposmia in more than six months.

Conclusion Rapid unexplicable weight gain and decreased olfactory perception in lupus may suggest neurolupus or NMDA encephalitis.

\section{PS10:184 CONSTRICTIVE PERICARDITIS AS THE FIRST PRESENTATION OF SYSTEMIC LUPUS ERYTHEMATOSUS: A CASE REPORT AND LITERATURE REVIEW}

${ }^{1} \mathrm{H}$ Jethwa, ${ }^{1} \mathrm{M}$ Rana, ${ }^{2} \mathrm{~J}$ Kitt, ${ }^{1} \mathrm{~J}$ Democratis, 'S Menzies, 'A Steuer, 'S Gindea. 'Wexham Park Hospital, Slough, UK; ${ }^{2}$ South Buckinghamshire Trust, South Buckinghamshire, UK

\subsection{6/lupus-2018-abstract.225}

Objective Although fibrinous and exudative pericarditis is a common feature of Systemic lupus erythematosus (SLE), found in $62 \%$ of lupus patients on autopsy, very few cases progress to (effusive) - constrictive pericarditis. We describe the unusual occurrence of constrictive pericarditis (CP) in a patient with Systemic Lupus Erythematosus.

Methods This is a chart review- based report of a lupus patient who had constrictive pericarditis as a presenting feature and a systematic literature review of previously published cases. We searched the English medical literature from 1963 to 2016 using PubMed, for terms: 'systemic lupus erythematosus' and 'constrictive pericarditis'.

Results A 49 year old African man presented with several weeks of malaise, weight loss, cough, breathlessness, peripheral oedema and hepatomegaly suggestive of right ventricular failure. An echocardiogram demonstrated features of effusive-constrictive pericarditis. The patient was initially treated for suspected tuberculosis; his symptoms progressed in spite of treatment. Further investigations confirmed positive lupus serology (ANA, anti-dsDNA $\mathrm{Ab}$ and anti-Sm Ab, low complement levels) and a raised urine protein: creatinine ratio of $177 \mathrm{mg} / \mathrm{mmol}$. A diagnosis of SLE was established and treatment with Hydroxychloroquine and Prednisolone was initiated. However, the $\mathrm{CP}$ was refractory to medical management eventually requiring Pericardiectomy.

A literature review identified six other cases of lupus patients with CP. Of these, four patients were male and average age was 38 years. CP was the presenting feature in four cases and TB was part of the differential diagnosis in five cases. The progression from exudative to constrictive pericarditis ranged from one week to six months. Pericardial biopsies performed in four cases showed non-specific chronic inflammation and fibrosis. CP resolved with corticosteroid treatment alone in one case; pericardectomy was necessary in the other five cases for symptom resolution.

Conclusions SLE should be included in differential diagnosis of constrictive pericarditis, especially in 'idiopathic' cases and in the context of poor response to tuberculosis treatment. Pericardial biopsy remains a crucial test in excluding an 